\title{
Application of a novel particle tracking algorithm in the flow visualization of an artificial abdominal aortic aneurysm
}

\author{
Yang Zhang ${ }^{\mathrm{a}}$, Yuan Wang, ${ }^{\mathrm{a},}$, Wenbo He ${ }^{\mathrm{a}}$ and Bin Yang ${ }^{\mathrm{b}}$ \\ ${ }^{a}$ Department of Fluid Machinery and Engineering, Xi'an Jiaotong University, Xianning Western Road \\ 28, Xi'an 710049, China \\ ${ }^{b}$ School of Chemical Engineering, Northwest University, Taibai Northern Road 229, Xi'an 710049, \\ China
}

\begin{abstract}
A novel Particle Tracking Velocimetry (PTV) algorithm based on Voronoi Diagram (VD) is proposed and briefed as VD-PTV. The robustness of VD-PTV for pulsatile flow is verified through a test that includes a widely used artificial flow and a classic reference algorithm. The proposed algorithm is then applied to visualize the flow in an artificial abdominal aortic aneurysm included in a pulsatile circulation system that simulates the aortic blood flow in human body. Results show that, large particles tend to gather at the upstream boundary because of the backflow eddies that follow the pulsation. This qualitative description, together with VD-PTV, has laid a foundation for future works that demand high-level quantification.
\end{abstract}

Keywords: Particle tracking velocimetry, Voronoi diagram, aortic blood flow, abdominal aortic aneurysm, pulsatile circulation system

\section{Introduction}

Velocity fields in aneurysms are usually highly unsteady and inhomogeneous in temporal and spatial dimensions because of the cyclic variations of pulsative flow. Laser Doppler Velocimetry was previously used to measure flow fields in aneurysm models [1]. However, this method is time consuming and has relatively high uncertainty [2]. Therefore, the acquisition of simultaneous flow fields in an entire plane is necessary. The high-speed imaging method Particle Tracking Velocimetry (PTV) is a suitable alternative. Similar to Particle Image Velocimetry (PIV), PTV tracks light-scattering small tracer particles seeded into the flow. However, PIV obtains a velocity field by a statistical displacement estimation of particle ensembles from an Eulerian viewpoint [3], whereas PTV tracks the instantaneous locations of all particles from a Lagrangian viewpoint. The resulting particle trajectories could lead to a more detailed analysis of flow structures.

\footnotetext{
${ }^{*}$ Corresponding author, Yuan Wang, Department of Fluid Machinery and Engineering, Xi'an Jiaotong University, Xianning Western Road 28, Xi'an 710049, China. Tel.: +86 2982663953; Fax: +86 2982668710; E-mail: wangyuan@mail.xjtu.edu.cn.
} 
Several studies have focused on PTV measurements in aneurysm models [4-6]. Most of these studies adopted PTV algorithms based on quasi-parallel motion [7] which is flawed by the uncertainty of selecting the interrogation radius Rs. As shown in Figure 1, PTV matches particles on two consecutive images that collectively describe a flow. The role of each particle on the first image is to select its matching candidates on the second image. It can be realized by three steps:

1. Placing this particle onto the second image with its coordinates $\left(x_{0}, y_{0}\right)$ unchanged;

2. Generating an interrogation area with $\left(x_{0}, y_{0}\right)$ and $R_{s}$, as colored grey in Figure 1;

3. Selecting the particles falling in this area as the candidates.

The interrogation radius $R_{S}$ is a parameter closely related to the specific structure of flow field. An effective value of $R_{s}$ should be no less than the genuine maximum inter-frame displacement, so that all particles are at least qualified to find their match. Choosing a reasonable value of $R_{s}$ faces two challenges: large spatial velocity gradient when there are two consecutive images and large temporal velocity fluctuation (such as pulsatile flow) when there are a sequence of images. Both challenges are highly common in aortic blood flow. However, to date, $R_{s}$ can only be roughly estimated before PTV starts to work. To solve this problem, this study proposes a new PTV algorithm free of $R_{s}$.

\section{VD-PTV and the test}

This new PTV involves three steps. The first step is to generate a characteristic curve for each particle on the image. The detailed procedure is shown in Figure 2. Figure 2(a) shows an original particle image. Figure 2(b) is the result of binarization and particle recognition of this image. Each recognized particle and its center are denoted by a white patch and a black dot, respectively. Figure 2(c) shows the division of this binarized image by Voronoi diagram (VD) [8]. The generated Voronoi polygons are marked by grey-scale colors. One of them is selected and highlighted (its edge is in bright color). As shown in Figure 2(d), this selected polygon is separated into a group of triangles (I, II, ...V) in a new Cartesian coordinate system, whose center $(\mathrm{x} 0, \mathrm{y} 0)$ coincides with the location of the original particle inside this polygon. As demonstrated in Figure 2(e), for a triangle marked as I [highlighted in panel (d)], the computation of the polar radius $r$ as a function of the polar angle $\alpha$ follows Eq. (1). After the rest of the triangles in Figure 2(d) (from II to V) undergo the same procedure, the selected polygon finally becomes a characteristic curve $\mathrm{r}(\alpha)$, as shown in Figure 2(f). The second step is the selection of candidates for all particles on the first image $\left\{x_{\mathrm{i}}\right\}$ by introducing a fixed interrogation radius $R_{s}$, as shown in Figure 1. The particle $x_{i}$ would have a group of matching candidates $\left\{y_{j}\right\}$. The third step is the successive comparison of the characteristic curves of all particles in $\left\{y_{j}\right\}$ with that of $x_{i}$. Any particle that corresponds to the highest curve similarity is selected as the final match of $x_{i}$.

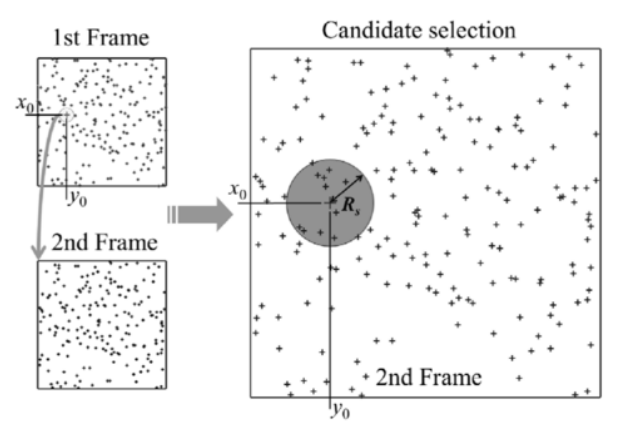

Fig. 1. Candidate selection in PTV. 

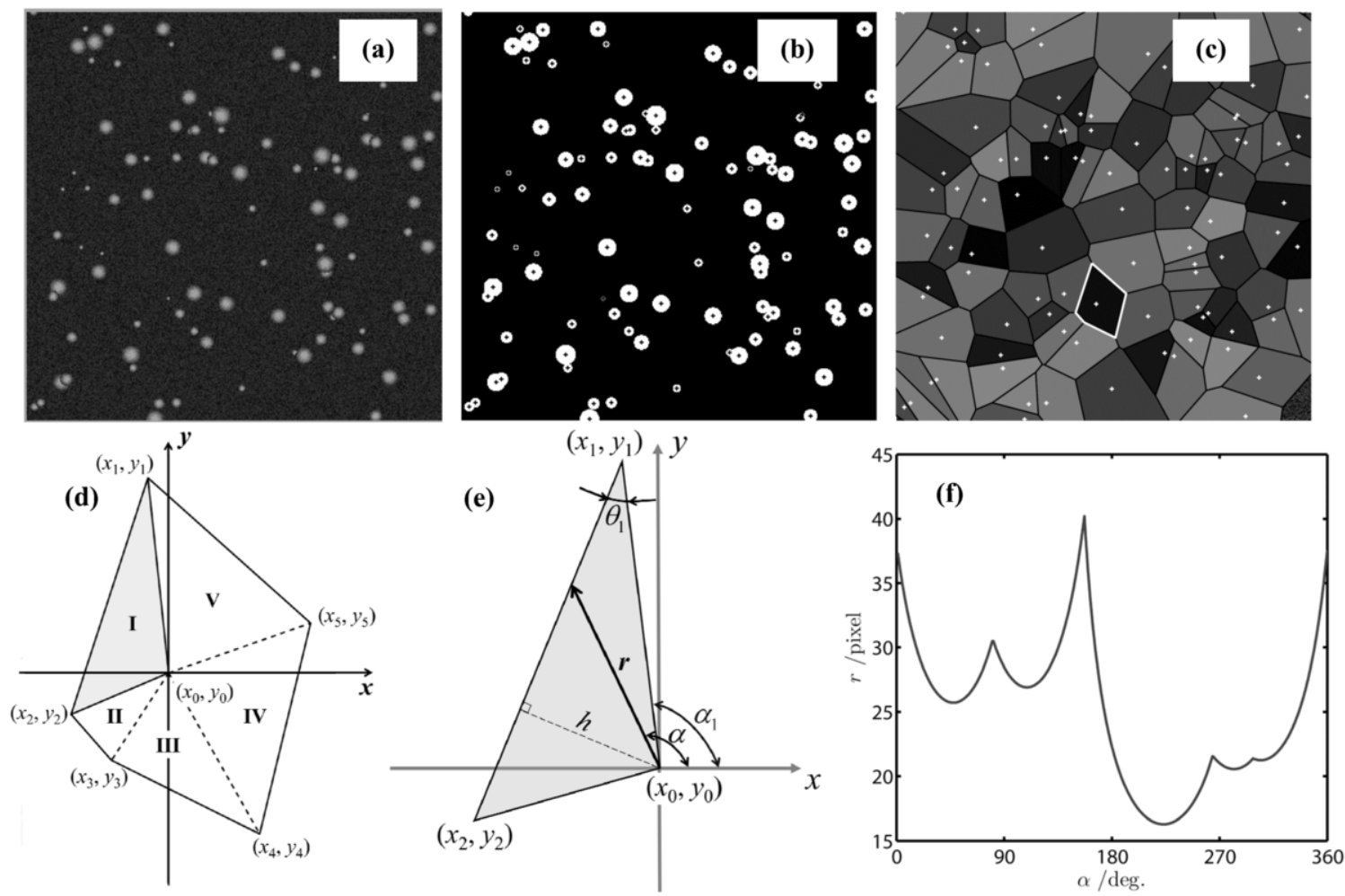

Fig. 2. From a Voronoi polygon to a characteristic curve: (a) an original particle image; (b) binarization and particle recognition of the image; (c) division of the binarized image into Voronoi polygons; (d) separation of the selected polygon into a group of triangles; (e) polar radius $r$ as a function of the polar angle $\alpha$ in triangle I; (f) after triangles from II to V undergo the same procedure as I, one can obtain a characteristic curve $r(\alpha)$ that corresponds to the selected polygon.

$$
\begin{aligned}
& \boldsymbol{r}(\boldsymbol{\alpha})=\frac{h}{\left|\sin \left(\boldsymbol{\alpha}+\theta_{1}-\alpha_{1}\right)+1-\operatorname{logical}\left(\theta_{1}-90^{\circ}\right)\right|} \quad \operatorname{logical}(x)= \begin{cases}1 & (x \neq 0) \\
0 & (x=0)\end{cases} \\
& h=\frac{\left|A x_{0}+B y_{0}+C\right|}{\sqrt{A^{2}+B^{2}}} \quad\left\{\begin{array}{l}
A=y_{2}-y_{1} \\
B=x_{1}-x_{2} \\
C=x_{2} y_{1}-x_{1} y_{2}
\end{array}\right.
\end{aligned}
$$

The computation of the similarity coefficient between two curves (particles) is specified in Figure 3. First, as shown in Figure 3(a), a pair of polygons that represent two particles are replaced by two characteristic curves $r_{1}$ and $r_{2}$, both normalized by $\left(\left|r_{1}\right|^{2}+\left|r_{2}\right|^{2}\right)^{1 / 2}$. To avoid the influence on the computation of similarity by inter-frame polygon rotation, $r_{1}$ is expanded twice (dotted), and $r_{2}$ (black) moves forward gradually, i.e., the phase difference $\alpha_{s}$ varies from 0 to $360^{\circ}$, thereby generating a group of instantaneous curves (grey). Each grey curve corresponds to a specific value of $\alpha_{s}$. For any one of the grey curves $r_{2}{ }^{\prime}\left(\alpha_{s}^{\prime}\right)$ (that is, $\left.\alpha_{s}=\alpha_{s}{ }^{\prime}\right)$, a similarity $C_{r}\left(\alpha_{s}{ }^{\prime}\right)$ exists between $r_{2}{ }^{\prime}\left(\alpha_{s}{ }^{\prime}\right)$ and the segment $r_{1}{ }^{\prime}=$ $r_{1}(\alpha)\left(\alpha \in\left[\alpha_{s}{ }^{\prime}, \alpha_{s}{ }^{\prime}+360^{\circ}\right]\right)$, and the computation of similarity follows Eq. (2), in which "cov" indicates the covariance of two vectors. After all grey curves are processed in the same way, a relation $C_{r}\left(\alpha_{s}\right)$ can be obtained, whose maximum value (white cycle) is adopted as the final similarity coefficient 

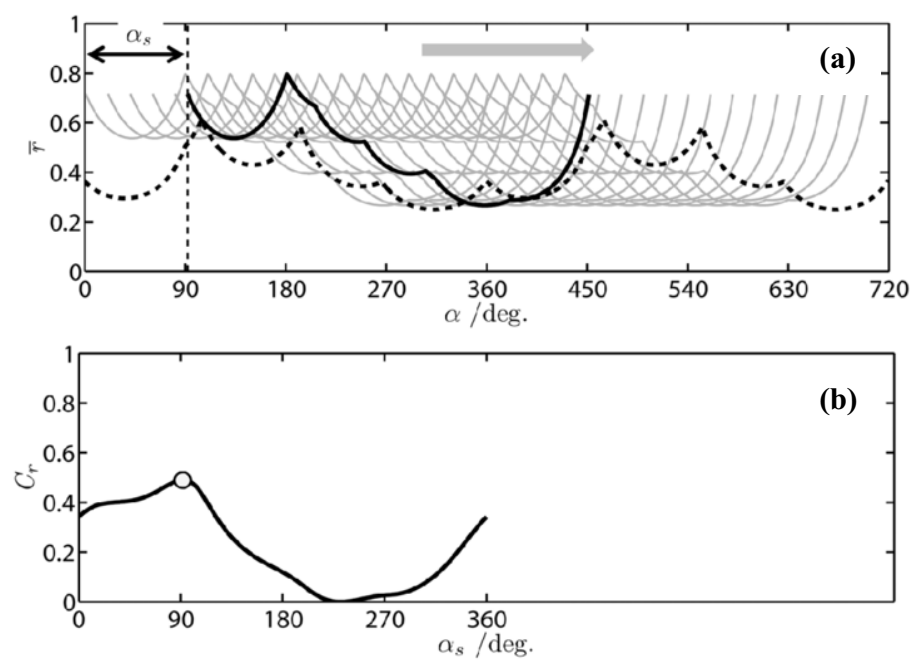

Fig. 3. Similarity coefficient between two polygons: (a) replacement of two polygons by characteristic curves $r_{1}$ (dotted) and $r_{2}$ (black). At $\alpha_{s}=\alpha_{s}^{\prime}, C_{r}\left(\alpha_{s}^{\prime}\right)$ indicates the similarity between $r_{2}^{\prime}\left(\alpha_{s}^{\prime}\right)$ and $r_{1}(\alpha)\left(\alpha \in\left[\alpha_{s}^{\prime}, \alpha_{s}{ }^{\prime}+360^{\circ}\right]\right)$.

between $r_{1}$ and $r_{2}$, as shown in Figure 3(b). Because the technique employs Voronoi diagram, this new PTV is named VD-PTV.

$$
C_{r_{1}, r_{2}}=\frac{\operatorname{cov}\left(r_{1}, r_{2}\right)}{\sqrt{\operatorname{cov}\left(r_{1}\right) \operatorname{cov}\left(r_{2}\right)}}
$$

An automated flow derived from numerical simulation [9], which is commonly used in the test of PTV algorithms, is adopted to test this new VD-PTV. It includes two consecutive particle images that collectively describe a complex flow (Figure 4(a)). Because the matching relations between the two images are known, users can compare them with their own results to evaluate the performance of the tested algorithms. The PTV algorithm DT-PTV is proven robust in dealing with high velocity gradients [7]. Hence, this algorithm is introduced as the counterpart of VD-PTV. The test result is shown in Figure 4(b). VD-PTV is capable of withstanding the influence of $R_{s}$ variation. Therefore, VD-PTV can fit for pulsative flow with fluctuating $R_{s 0}$-the genuine maximum inter-frame displacement-by adopting a sufficiently large $R_{s}$.

\section{Application of VD-PTV in an artificial AAA system}

An in vitro experiment was performed to study the flow structure in an artificial AAA system. As shown in Figure 5, the circulation system is composed of a centrifuge pump and two solenoid valves; the pump generates the flow, and the valves simulate the pulsating waveforms [10] of an aortic blood flow. The function of the valves is regulated by a couple of compliances and an after load (a flow regulator) [11].

An electromagnetic flowmeter was introduced to measure the flow rate of the entire system, and a pressure sensor was used to measure the pulsation pressure in the test chamber with a maximum 


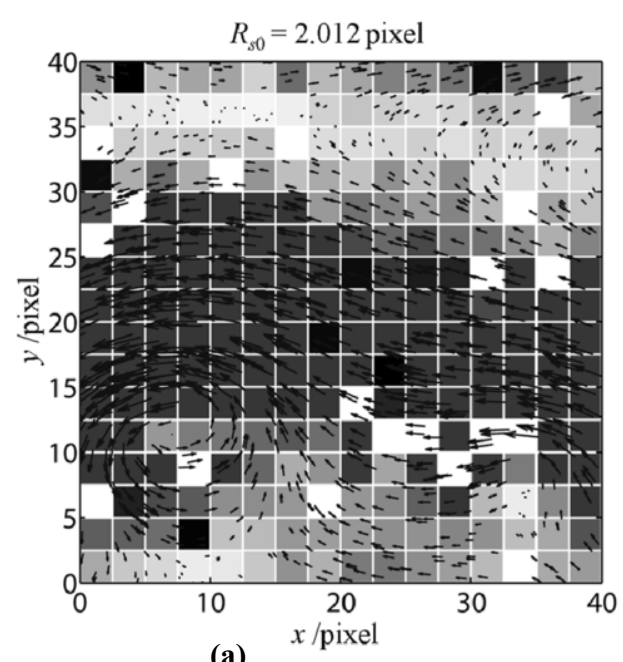

(a)

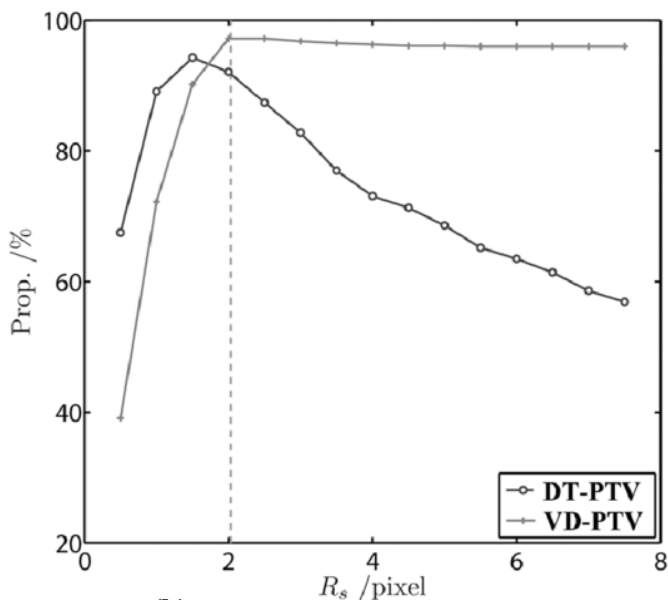

(b)

Fig. 4. Test for PTV: (a) test flow, where inter-frame matches are denoted by black errors, and darker area indicates stronger velocity gradient; (b) test results, where dashed line corresponds to $R_{s 0}$, i.e., the genuine maximum inter-frame displacement.

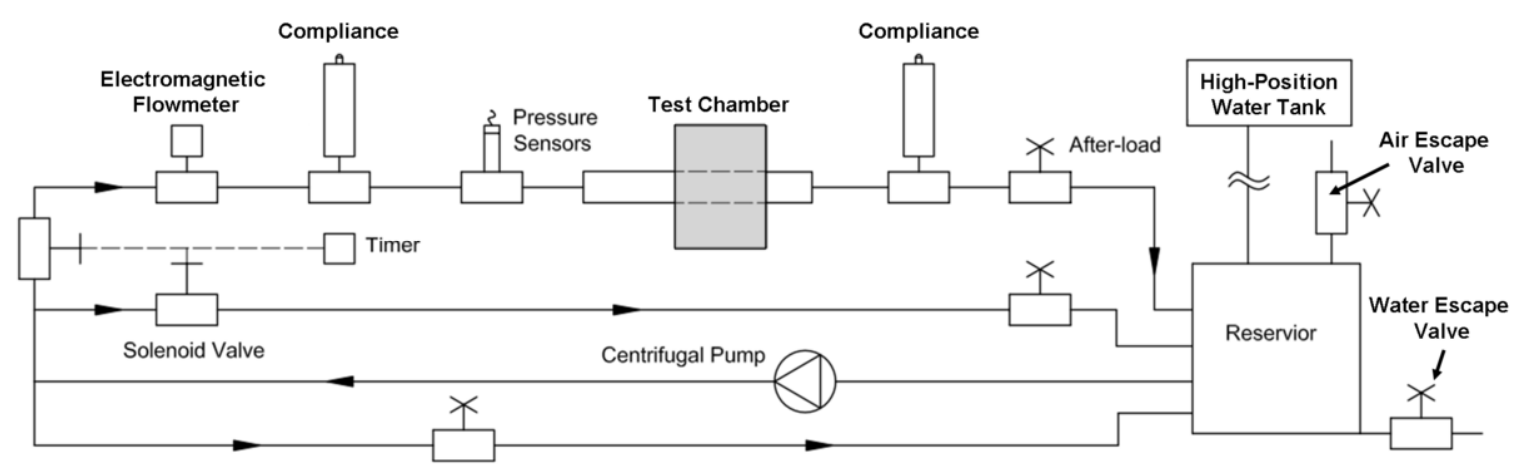

Fig. 5. Schematic of the circulation system.

sample frequency of $500 \mathrm{~Hz}$ and a measuring rage $[-40,250]$ in $\mathrm{mm} \mathrm{Hg}$. The Artificial model placed in the test chamber was made of transparent PMMA material (Figure 6(a)). The liquid was a mixture composed of $45 \%$ distilled water and $55 \%$ glycerine, served as artificial blood [2]. Specifics of the setup above can be found in [12].

The artificial blood was seeded with fluorescent tracer particles with a mean diameter of approximately $10 \mu \mathrm{m}$ and a density of $1500 \mathrm{~kg} / \mathrm{m}^{3}$. The particles were labeled with Rhodamine 610 with a maximum emission at $584 \mathrm{~nm}$. A high-speed laser generator $(100 \mathrm{~mJ}, 100 \mathrm{~Hz})$ was applied to form a light sheet in the middle of the test chamber along the main stream direction. A CCD camera was used to record the particle motion on the sheet from side view with a frequency of $10 \mathrm{~Hz}$, i.e., ten times larger than that of the generated pulsatile flow and that of the aortic blood flow in human body. One of the twenty recorded pulsating periods was applied to demonstrate the effectiveness of VD-PTV.

VD-PTV can easily resist the drastic changes in $R_{s}$. Hence, a fixed value $R_{s}=L / 2$ was applied to address this period with a pulsation, whose resulting inter-frame displacement is hard to predict quantitatively. $L=80 \mathrm{~mm}$ refers to the entire length of the AAA chamber. As a result, VD-PTV with a 

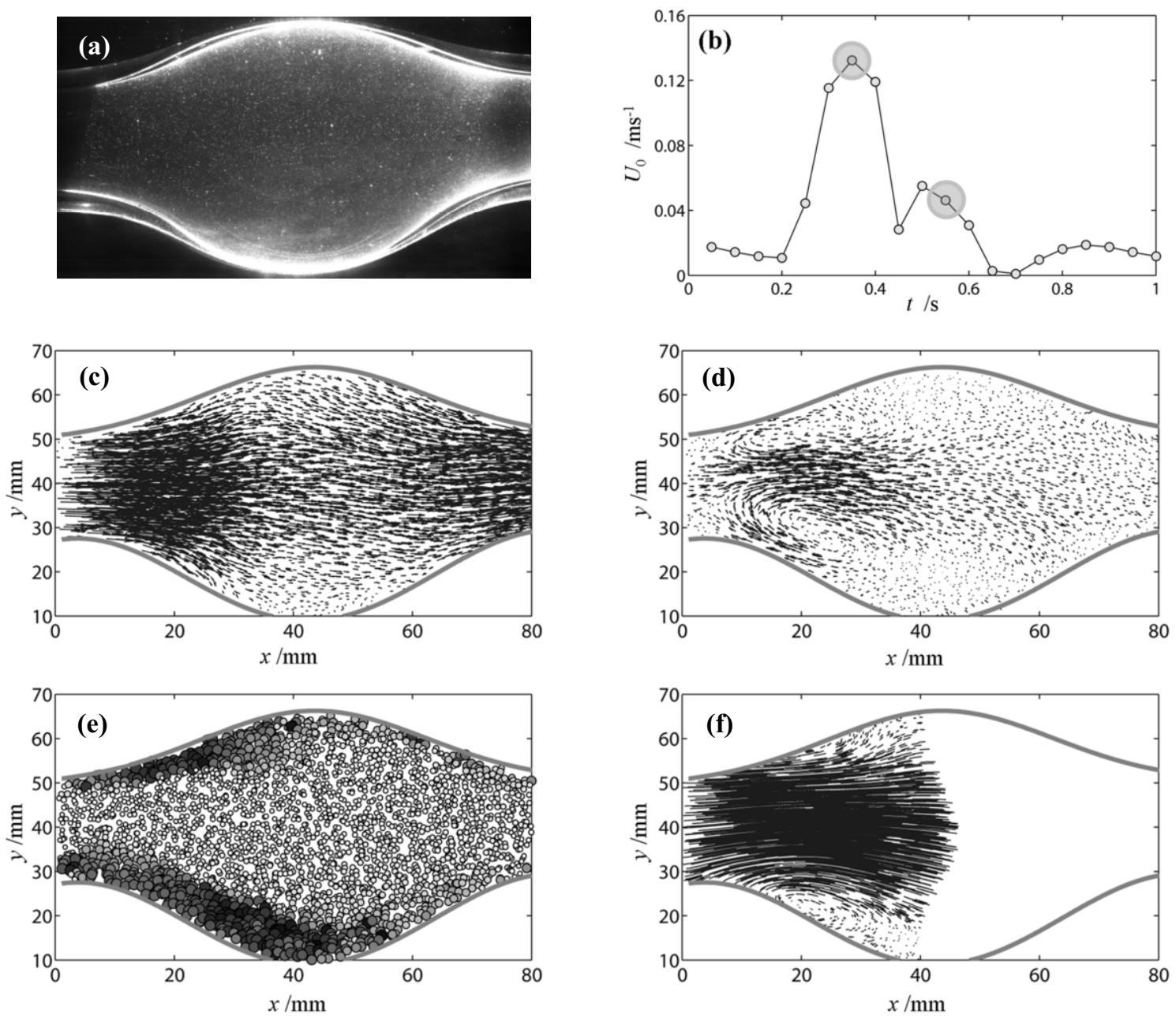

Fig. 6. Flow visualization in the artificial AAA chamber: (a) original image at $t=0.05 \mathrm{~s}$; (b) reference flow velocity $U_{0}$ along the central line $(y=40 \mathrm{~mm}$ ) as a function of $t$; (c) match result (velocity field) by VD-PTV at $t=0.35 \mathrm{~s}$, which is based on the information of particle location at $t=0.35 \mathrm{~s}$ and $t=0.4 \mathrm{~s}$; (d) match result at $t=0.55 \mathrm{~s}$; (e) particle distribution at $t=0.55 \mathrm{~s}$, where darker color denotes stronger reverse velocity, and larger particle (idealized as absolute circle) indicates greater diameter; (f) particle trajectories in the upstream part of the chamber during the period $t=0.4 \sim 0.6 \mathrm{~s}$.

sufficiently large $R_{s}$ can successfully visualize the pulsation (Figure 6(c), corresponding to the left highlighted circle in Figure 6(b)) and the subsequent recirculation (Figure 6(d), corresponding to the right highlighted circle in Figure 6(b)). From a Lagrangian viewpoint, PTV can also accurately track the information carried by the particles. Figure 6(e) shows the instantaneous particle distribution corresponding to the instantaneous flow field in Figure 6(d). Together with the result of particle trajectories (Figure 6(f)), it indicates that the upstream boundary is the gathering place of large particles that move against the stream, thereby bearing extra body force and reverse shear compared with the other areas in the chamber. Such conclusion can hardly be obtained by traditional PIV analysis. 


\section{Conclusion}

The proposed VD-PTV is effective in addressing pulsatile flows that contain great temporal velocity gradients. It can yield qualitative yet convincing results from an artificial AAA chamber, such as the mass distribution and particle trajectories in the chamber. In future studies, the algorithm will be further applied in an in vitro system with rigorously regulated similarity criteria, and is anticipated to provide quantitative evidences.

\section{Acknowledgement}

This work is funded by Natural Science Foundation of China $(11272252,11102153)$ and China Postdoctoral Science Foundation (2014M552443).

\section{References}

[1] R. Bordás, S. Seshadhri, G. Janiga et al., Experimental validation of numerical simulations on a cerebral aneurysm phantom model, Intervention in Medicine and Applied Sciences 4 (2012), 193-205.

[2] C. Roloff, R. Bordás, R. Nickl et al., Investigation of the velocity field in a full-scale model of a cerebral aneurysm, International Journal of Heat and Fluid Flow 43 (2013), 212-219.

[3] G. Liu, Y. Zhang, H. Chen et al., Flow visualization in the outflow cannula of an axial blood pump, Bio-Medical Materials Engineering 24 (2014), 117-122.

[4] T.M. Liou and S.N. Liou, Pulsatile flows in a lateral aneurysm anchored on a stented and curved parent vessel, Experimental Mechanics 44 (2004), 253-260.

[5] E. Boutsianis, M. Guala, U. Olgac et al., CFD and PTV steady flow investigation in an anatomically accurate abdominal aortic aneurysm, Journal of Biomechanical Engineering 131 (2009), 011008.

[6] C. Stamatopoulos, D. Mathioulakis, Y. Papaharilaou et al., Experimental unsteady flow study in a patient-specific abdominal aortic aneurysm model, Experiments in Fluids 50 (2011), 1695-1709.

[7] Y. Zhang, Y. Wang and P. Jia, Improving the Delaunay tessellation particle tracking algorithm in the three-dimensional field, Measurement 49 (2014), 1-14.

[8] F. Aurenhammer, Voronoi Diagrams-A Survey of a Fundamental Geometric Data Structure, ACM Computing Surveys 23 (1991), 345-405.

[9] K. Okamoto, S. Nishio, T. Kobayashi et al., Evaluation of the 3D-PIV standard images, Journal of Visualization 3 (2000), 115-123.

[10] S.I. Bernad, A. Bosioc, E.S. Bernad and M.L. Craina, Comparison between experimentally measured flow patterns for straight and helical type graft, Bio-Medical Materials Engineering 24 (2014), 853-860.

[11] C.K. Chong and T.V. How, Flow patterns in an endovascular stent-graft for abdominal aortic aneurysm repair, Journal of Biomechanics 37 (2004), 89-97.

[12] W.B. He, Y. Wang and X.Y. Gong, Influences of the compliances and the resistance on pulsatile flow waveforms, Applied Mechanics and Materials 477 (2013), 217-220. 